\title{
Velocity-space sensitivities of neutron emission spectrometers at the tokamaks JET and ASDEX upgrade in deuterium plasmas
}

Jacobsen, A.S.; Binda, F.; Cazzaniga, C.; Eriksson, J.; Hjalmarsson, A.; Nocente, M.; Salewski, Mirko; Tardini, G.

Published in:

Review of Scientific Instruments

Link to article, DOI:

$10.1063 / 1.4991651$

Publication date:

2017

Document Version

Peer reviewed version

Link back to DTU Orbit

Citation $(A P A)$ :

Jacobsen, A. S., Binda, F., Cazzaniga, C., Eriksson, J., Hjalmarsson, A., Nocente, M., Salewski, M., \& Tardini, G. (2017). Velocity-space sensitivities of neutron emission spectrometers at the tokamaks JET and ASDEX upgrade in deuterium plasmas. Review of Scientific Instruments, 88(7), [073506]. https://doi.org/10.1063/1.4991651

\section{General rights}

Copyright and moral rights for the publications made accessible in the public portal are retained by the authors and/or other copyright owners and it is a condition of accessing publications that users recognise and abide by the legal requirements associated with these rights.

- Users may download and print one copy of any publication from the public portal for the purpose of private study or research.

- You may not further distribute the material or use it for any profit-making activity or commercial gain

- You may freely distribute the URL identifying the publication in the public portal 


\title{
Velocity-space sensitivities of neutron emission spectrometers at the tokamaks JET and ASDEX Upgrade in deuterium plasmas
}

\author{
A. S. Jacobsen, ${ }^{1, a)}$ F. Binda, ${ }^{2}$ C. Cazzaniga, ${ }^{3}$ J. Eriksson, ${ }^{2}$ A. Hjalmarsson, ${ }^{2}$ \\ M. Nocente, ${ }^{4}$ M. Salewski, ${ }^{5}$ G. Tardini, ${ }^{1}$ JET contributors, ${ }^{\text {b) }}$ and the ASDEX Upgrade \\ Team $^{1, c)}$
}

(EUROfusion Consortium, JET, Culham Science Centre, Abingdon, OX14 3DB, UK)

1) Max-Planck-Institut für Plasmaphysik, Garching, Germany

${ }^{2)}$ Department of Physics and Astronomy, Uppsala University,

Sweden

3) ISIS Facility, Science and Technology Facilities Council, Rutherford Appleton Laboratory, Didcot, OX11 0QX, UK

4) Dipartimento di Fisca 'G. Occhialini', Università degli Studi di Milano-Bicocca, Milano, Italy

5) Technical University of Denmark, Department of Physics, Kgs. Lyngby, Denmark

Future fusion reactors are foreseen to be heated by the energetic alpha particles produced in fusion reactions. For this to happen, it is important that the energetic ions are sufficiently confined. In present day fusion experiments, energetic ions are primarily produced using external heating systems such as neutral beam injection and ion cyclotron resonance heating. In order to diagnose these fast ions, several different fast-ion diagnostics have been developed and implemented at the various experiments around the world. The velocity-space sensitivities of fast-ion diagnostics are given by so-called weight functions. Here instrument-specific weight functions are derived for neutron emission spectrometry detectors at the tokamaks JET and ASDEX Upgrade, for the $2.45 \mathrm{MeV}$ neutrons produced in deuterium-deuterium reactions in deuterium plasmas. Using these it is possible to directly determine which part of velocity space each detector observes.

\footnotetext{
a) Electronic mail: asger.jacobsen@ipp.mpg.de

b) See the author list of "Overview of the JET results in support to ITER" by X. Litaudon et al. to be published in Nuclear Fusion Special issue: overview and summary reports from the 26th Fusion Energy Conference (Kyoto, Japan, 17-22 October 2016)

c) For a list of team members, see appendix of H. Zohm et al. ${ }^{40}$ 


\section{INTRODUCTION}

Several different types of diagnostics are used to measure fast ions in fusion plasmas. Each diagnostic observes a certain part of the fusion plasma, given by its orientation relative to the plasma. In addition to these spatial observation regions, every fast-ion diagnostic instrument measures only certain parts of the velocity space of the fast ions. This is determined by the velocity-space sensitivity functions, so-called fast-ion velocity-space weight functions, $w$. These weight functions have now been developed for many of the most common fast-ion diagnostics: collective Thomson scattering ${ }^{1}$, fast-ion $\mathrm{D}_{\alpha}$ spectroscopy $^{2,3}$, neutral particle analyzers $^{2,4}$, neutron emission spectrometry (NES) $)^{5,6}$ and gamma ray spectroscopy ${ }^{7,8}$. Here we focus on NES weight functions and account for the instrumental response functions of the different detectors. NES weight functions relating neutron energy spectra to velocity space have been developed $i^{6}$. These fundamental NES weight functions have proven useful in the analysis of NES measurements ${ }^{9,10}$. Taking into account the instrument-specific response function of a given diagnostic instrument, it becomes possible to calculate instrument-specific weight functions that directly relate the given measurements to the velocity space of the fast ions ${ }^{5}$. Here, such instrument-specific weight functions are calculated for three of the neutron spectrometers at JET and the spectrometer at ASDEX Upgrade. These allow a direct comparison of the velocity-space sensitivities of the different types of detectors. Our calculations and results are directly applicable to neutron emission spectrometers installed on other machines as long as the instrumental response functions are available. These include time-of-flight spectrometers on EAST ${ }^{11}$ and $\mathrm{LHD}^{12}$, and compact spectrometers on $\mathrm{EAST}^{13}$, FTU $^{14}$ and TCV.

This paper is organized as follows. In section II the different neutron emission spectrometers most commonly used for measuring D-D neutrons at JET and the spectrometer at ASDEX Upgrade are described. Section III describes the underlying fundamental neutron energy NES weight functions. In section IV the instrument-specific weight functions are calculated for the different neutron spectrometers, and the observable regions in velocity space are presented for each. The results are discussed in section $\mathrm{V}$ and conclusions are summarized in section VI. 


\section{JET AND ASDEX UPGRADE NEUTRON EMISSION SPECTROMETERS}

Several neutron emission spectrometers are installed at $\mathrm{JET}^{15-19}$. One of these is the time-of-flight spectrometer TOFOR ${ }^{15}$. TOFOR consists of two sets of detectors placed at a known distance from each other. By comparing the time-traces of neutron interactions in these detectors, it is possible to construct a time-of-flight spectrum, which reflects the energy distribution of the incoming neutrons. TOFOR is placed in the roof laboratory at JET viewing the plasma from above with a line-of-sight perpendicular to the magnetic field, as is shown in the left part of figure 1, which shows a side-view of JET including TOFOR and the magnetic proton recoil (MPR) deuterium-tritium neutron emission spectrometer ${ }^{18}$. The right part of figure 1 shows a top view of JET with the lines-of-sight of TOFOR and the MPR spectrometers indicated in blue and magenta, respectively.
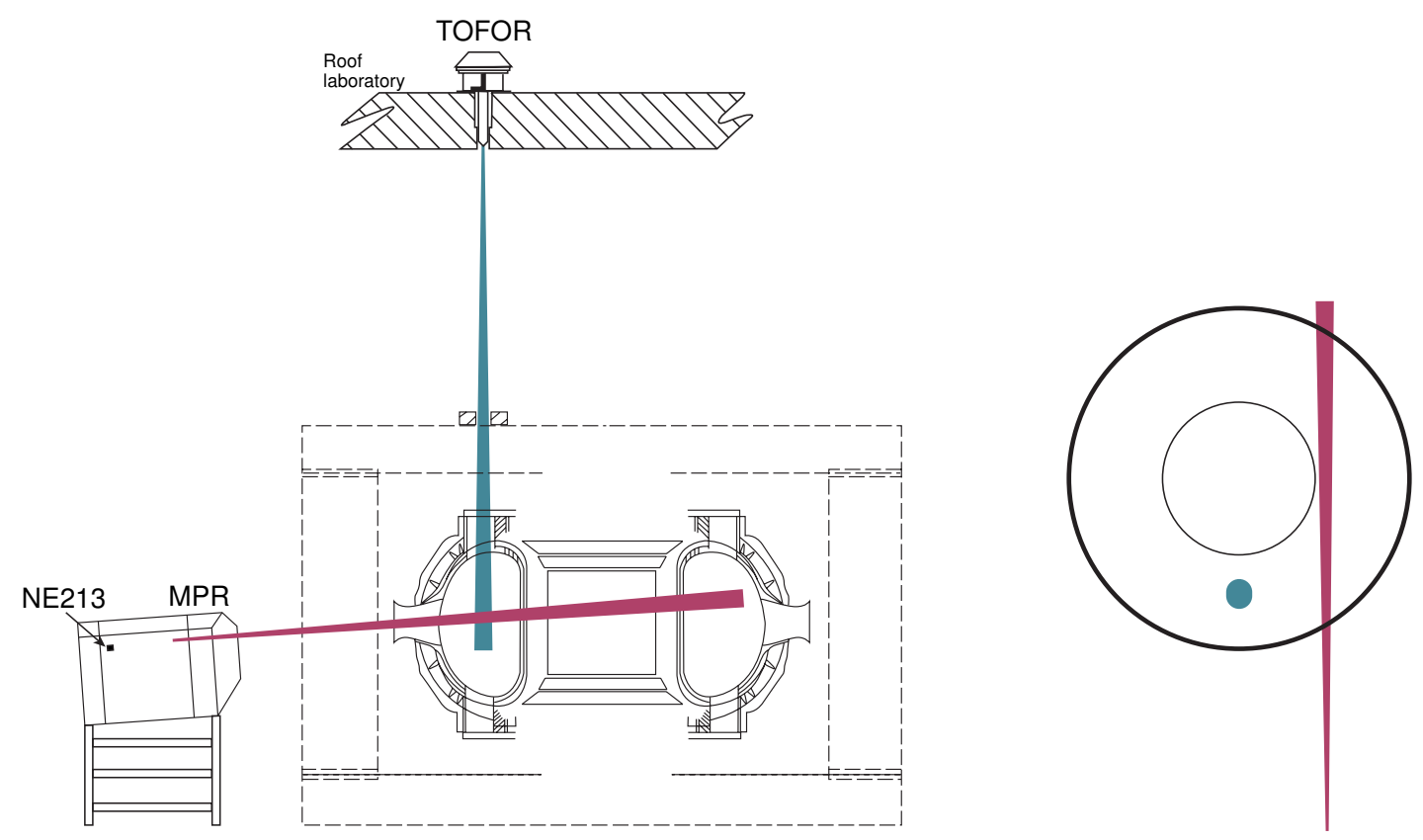

FIG. 1: Sketch of the locations of the NES spectrometers at JET.

The other two JET neutron energy spectrometers considered here are compact detectors. One of them uses the liquid organic scintillator material NE213 to detect neutrons ${ }^{16,20}$. A neutron can excite the scintillator material through neutron-hydrogen elastic scattering events. The scintillator emits light in the subsequent relaxation. The emitted light depends 
on the energy of the incoming neutron. The unit of the light output of the NE213 spectrometer is MeVee (megaelectronvolt electron equivalent), which is the light output that would be produced by an electron with that energy. This unit is chosen since the light output response to incoming electrons is linear ${ }^{21}$. The compact NE213 spectrometer is placed in the back of the MPR spectrometer and shares its line-of-sight. The angle between the magnetic field and the line-of-sight of the NE213 detector when it intersects the plasma centre is about $45^{\circ}$. The third neutron energy spectrometer uses a synthetic single-crystal diamond to detect neutrons through elastic scattering of the neutrons on ${ }^{12} \mathrm{C}$ nuclei ${ }^{17,22}$. This spectrometer is located behind the NE213 spectrometer and thus shares the same line-of-sight.

A compact NES spectrometer based on the organic scintillator material BC501A is installed at ASDEX Upgrade ${ }^{23,24}$. The detector views the plasma horizontally with a line of sight perpendicular to the magnetic field. The detection principle is identical to that of the NE213 JET detector.

Instrumental response functions describe the signal which a detector will produce when measuring neutrons of a certain energy. They are needed for relating measured signals to neutron energies. The instrumental response function of TOFOR is shown in figure $2 \mathrm{a}$. The TOFOR response function consists of two parts. The first is the neutron scattering in the detector which has been calculated using a Monte Carlo code, the second is an empirically determined broadening caused by the scintillator and electronics response ${ }^{15}$. The energy resolution is about 7 percent, which means that there is almost a 1:1 correspondence between the neutron energy and the time-of-flight, i.e. a measured flight time corresponds to a welldefined neutron energy. The low intensity spread towards long flight times is due to neutrons scattering multiple times in the detectors.

Figures $2 \mathrm{~b}-2 \mathrm{~d}$ show the response functions of the three compact detectors considered here. They are very different from that of TOFOR. For a given light output value or deposited energy there exists a minimum neutron energy threshold which the neutrons must exceed in order to create that signal. However, neutrons with higher energies can create the same response in the instrument as well. Nevertheless, the observable region in velocity space is still restricted for large light output and deposited energy values as will be shown in section IV. The instrumental response functions of the compact spectrometers are calculated by a Monte Carlo simulation of the detector response to mono-energetic neutrons. The simulated detector response is fitted to experimental measurements of mono-energetic neutrons for 


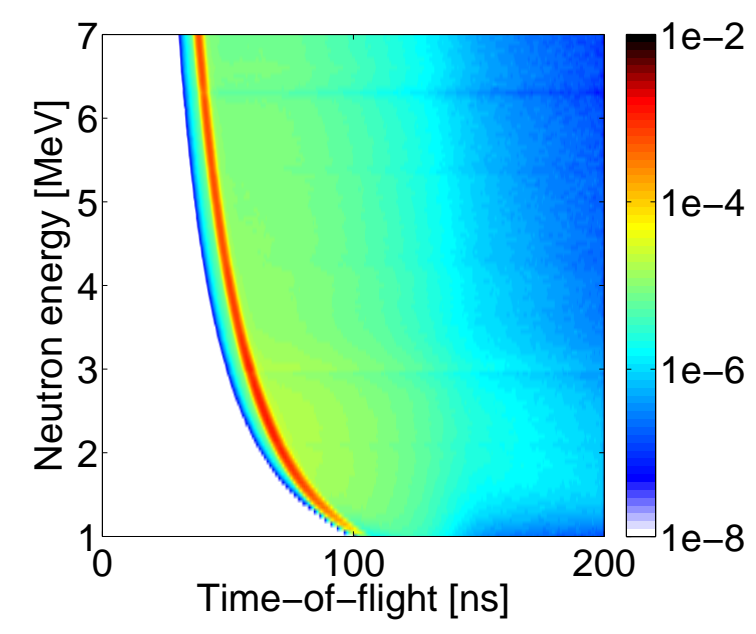

(a) TOFOR.

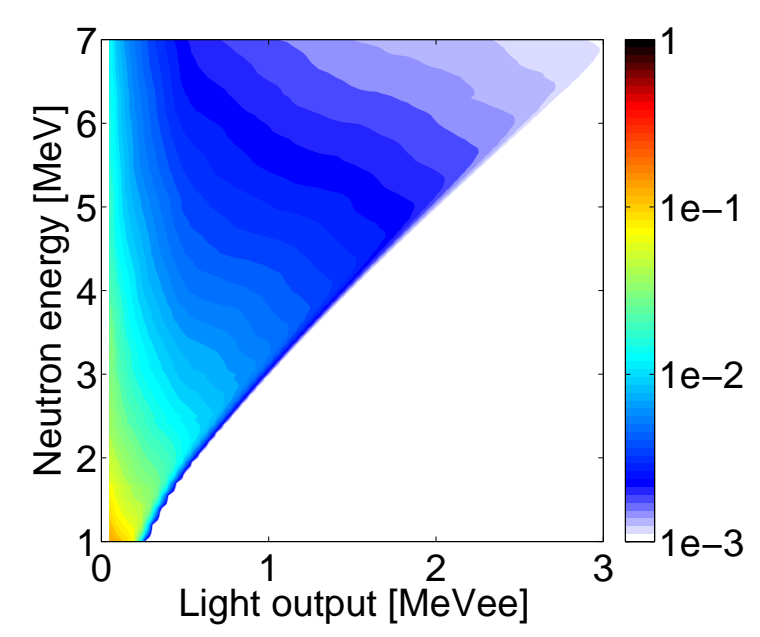

(c) NE213.

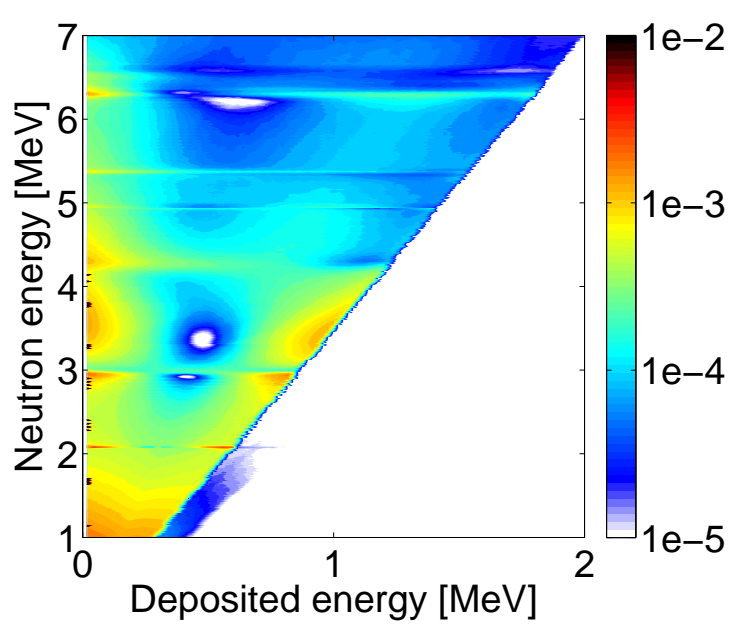

(b) Diamond.

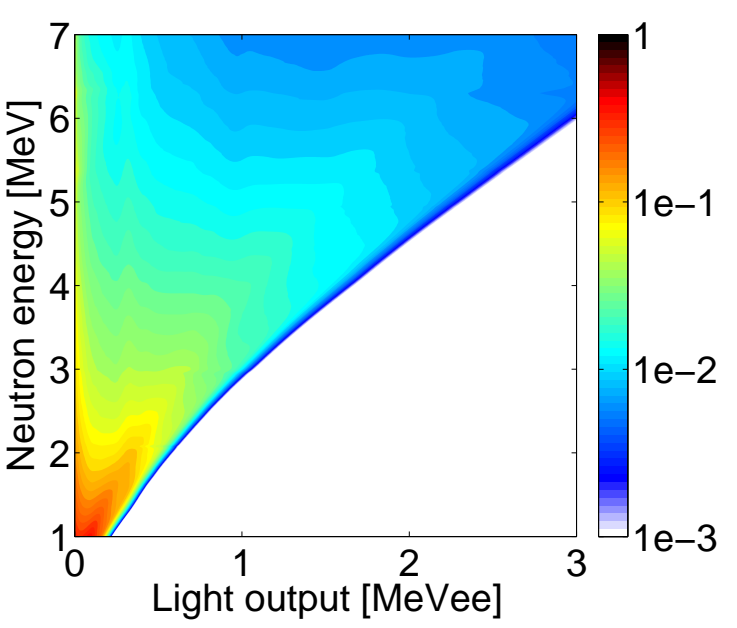

(d) BC501A.

FIG. 2: Instrumental response functions for the three most commonly used neutron spectrometers at JET and the ASDEX Upgrade detector.

several different energies. To fit the measurements, the simulated spectra need to be folded with a Gaussian describing the instrumental broadening ${ }^{16,17,25,26}$. In the case of the NE213 detector a mono-energetic neutron source was not available. Instead measurements obtained with another NE213 detector of similar size was used ${ }^{27}$.

Figure $2 \mathrm{~b}$ shows the response function of the diamond detector. The threshold neutron energy is given by $3.52 \times E_{d e p}$, where $E_{d e p}$ is the deposited energy. This can be derived from conservation of energy and momentum of the elastic scattering of a neutron on a ${ }^{12} \mathrm{C}$ atom. The diamond response function contains several stripe-like features and local minima. 
These are caused by the angular dependence of the cross section of this elastic scattering. Comparing figures $2 \mathrm{c}$ and $2 \mathrm{~d}$, it is clear that the instrumental response functions of the NE213 and BC501A detectors are rather different, even though the two detectors are based on identical detection principles. This is caused by the different detector sizes, geometries, scintillator-to-photomultiplier coupling and other differences in the detailed designs.

\section{NES NEUTRON ENERGY WEIGHT FUNCTIONS}

Before the instrument-specific weight functions can be calculated, the fundamental neutron energy weight functions are needed. They are briefly described here. For a comprehensive derivation and discussion of these, see reference ${ }^{6}$.

The relation between a neutron energy spectrum, $s_{E_{n}}\left(E_{n, 1}, E_{n, 2}, \phi\right)$, and the fast-ion distribution function, $f(E, p, \mathbf{r})$ is given by

$$
s_{E_{n}}\left(E_{n, 1}, E_{n, 2}, \phi\right)=\iiint w_{E_{n}}\left(E_{n, 1}, E_{n, 2}, \phi, E, p, \mathbf{r}\right) f(E, p, \mathbf{r}) \mathrm{d} E \mathrm{~d} p \mathrm{~d} \mathbf{r}
$$

where $w_{E_{n}}\left(E_{n, 1}, E_{n, 2}, \phi, E, p, \mathbf{r}\right)$ is the fundamental neutron energy weight function. It can be written as

$$
\begin{aligned}
w_{E_{n}}\left(E_{n, 1}, E_{n, 2}, \phi, E, p, \mathbf{r}\right)= & R(\phi, E, p, \mathbf{r}) \\
& \times \int_{E_{n, 1}}^{E_{n, 2}} \operatorname{pdf}_{E_{n}}\left(E_{n}, \phi, E, p\right) \mathrm{d} E_{n},
\end{aligned}
$$

where $R(\phi, E, p, \mathbf{r})$ is the rate of neutrons emitted towards the detector per fast ion irrespective of the energy of the neutrons and $\operatorname{pdf}_{E_{n}}\left(E_{n}, \phi, E, p\right)$ is the probability density function of the neutron energy. $E_{n}$ is the neutron energy, $\phi$ is the angle between the line-of-sight of the detector and the magnetic field, $E$ and $p$ are the energy and pitch of the fast ions, respectively, and $\mathbf{r}$ holds the spatial coordinates. The pitch is defined as $p=\frac{v_{\|}}{v}$, where $v_{\|}$is the velocity parallel to the magnetic field and $v$ is the magnitude of the velocity of the fast ions.

Figure 3 shows the neutron rate per fast ion, $R$, from equation (2), calculated numerically for $\phi=45$ and $\phi=90$ as explained in $^{6}$. They are plotted as functions of the energy and pitch of the fast ions. All examples in this paper are shown for ion energies up to $5 \mathrm{MeV}$, relevant for plasmas heated by ion cyclotron resonance heating. Our formalism applies just 
as well to lower energies typical for neutral beam injection. The neutron rate increases with energy as is expected as the D-D fusion cross section increases with energy in this energy range. Furthermore, a very clear pitch dependence, which is different for the two $\phi$-angles, is observed. This comes from the angular dependence of the fusion cross section, and from the fact that a neutron is more likely to be created moving in the same direction as the center of mass of the reactants.

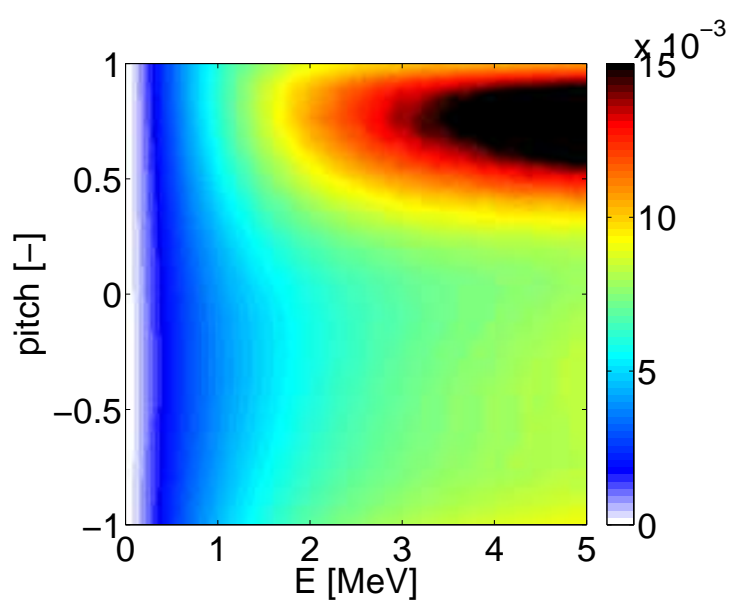

(a) $\phi=45^{\circ}$.

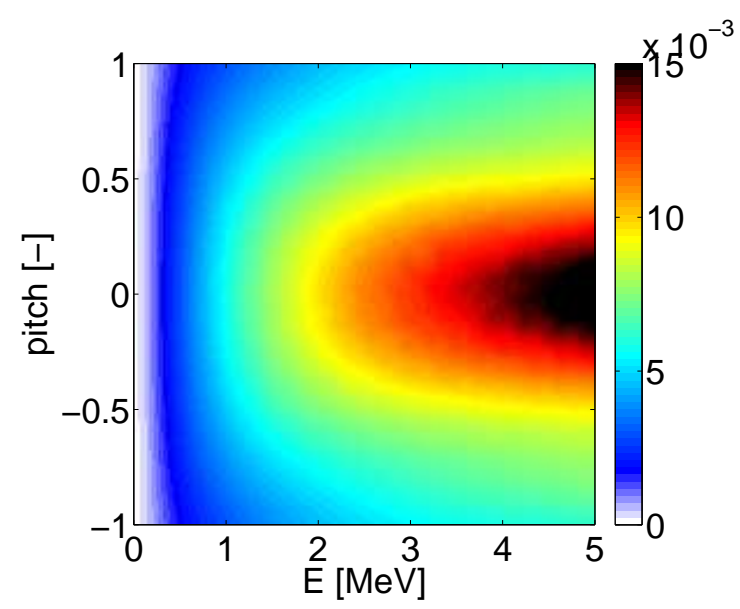

(b) $\phi=90^{\circ}$.

FIG. 3: The rate part, $R$, of NES energy weight functions calculated numerically for two different $\phi$-angles in units of neutrons per second per fast ion, calculated for a thermal ion density of $5 \times 10^{19} \mathrm{~m}^{-3}$. The pitch is defined as $\frac{v_{\|}}{v}$ where $v_{\|}$is the velocity parallel to the magnetic field and $v$ is the magnitude of the velocity of the fast ions.

Figure 4 shows examples of the probability part of NES neutron energy weight functions from equation (2) calculated for neutron energies of $2 \mathrm{MeV}, 4 \mathrm{MeV}$ and $6 \mathrm{MeV}$ and $\phi$-angles of $45^{\circ}$ and $90^{\circ}$. An energetic ion placed in the coloured region can produce a neutron with the given energy observed at the angle $\phi$ in a fusion reaction with a thermal ion. Ions in the white regions cannot create detectable neutrons at the given neutron energy. The shapes of these functions are determined by conservation of energy and momentum.

The complete NES neutron energy weight functions are the products of figures 3 and 4 according to equation (2). These are shown in figure 5. These fundamental NES weight functions show how localized a velocity-space sensitivity an NES instrument can at best obtain. 


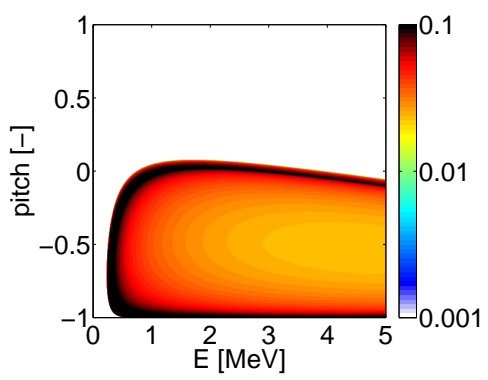

(a) $E_{n}=2 \mathrm{MeV}, \phi=45^{\circ}$.

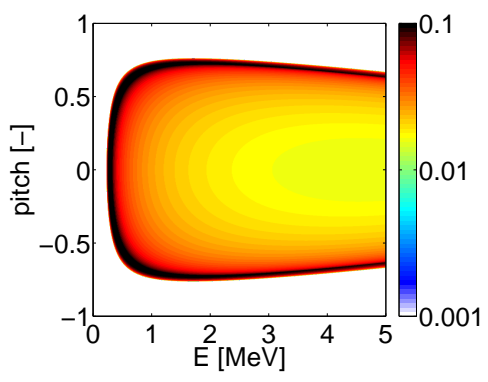

(d) $E_{n}=2 \mathrm{MeV}, \phi=90^{\circ}$.

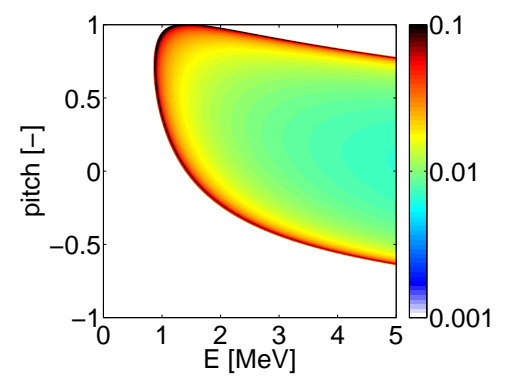

(b) $E_{n}=4 \mathrm{MeV}, \phi=45^{\circ}$.

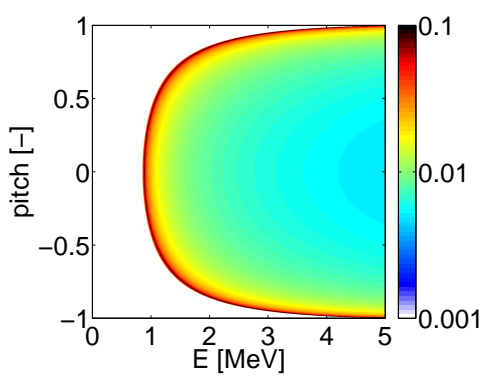

(e) $E_{n}=4 \mathrm{MeV}, \phi=90^{\circ}$.

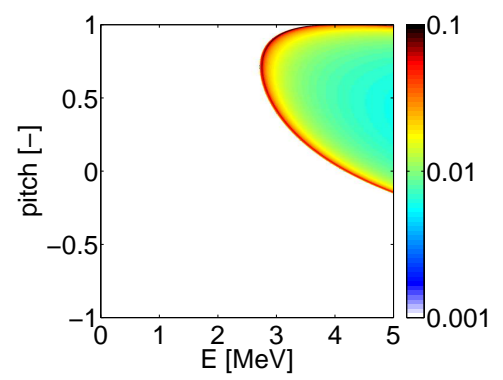

(c) $E_{n}=6 \mathrm{MeV}, \phi=45^{\circ}$.

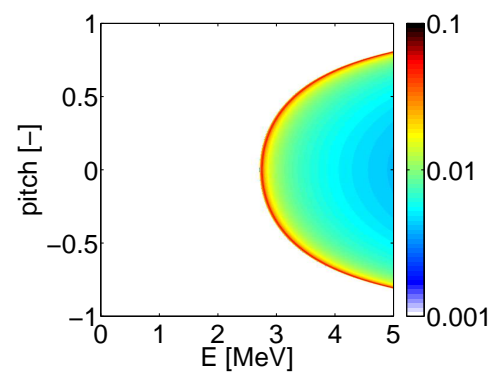

(f) $E_{n}=6 \mathrm{MeV}, \phi=90^{\circ}$.

FIG. 4: The probability part of NES neutron energy weight functions calculated analytically using the equations from reference ${ }^{6}$ for a neutron energy bin width of $50 \mathrm{keV}$.

(a)-(c) show examples calculated for a $\phi$-angle of $45^{\circ}$ corresponding to the oblique

line-of-sight of the two compact spectrometers at JET considered here. (d)-(f) show examples calculated for a radial view corresponding to TOFOR and the ASDEX Upgrade spectrometer.

\section{INSTRUMENT-SPECIFIC NES WEIGHT FUNCTIONS}

As explained in section III the neutron energy weight functions relate the sought-after fast-ion distribution function to the neutron energy spectra. However, real neutron detectors do not directly measure the neutron energy. Instead they measure a derived quantity as illustrated by the instrumental response functions in figure 2. The three detectors at JET measure time-of-flight in ns, light output in MeVee and deposited energy in $\mathrm{MeV}$, respectively. Thus it is beneficial to relate directly the measured signal to the fast-ion distribution function:

$$
s\left(x_{1}, x_{2}, \phi\right)=\iiint w_{\text {instrument }}\left(x_{1}, x_{2}, \phi, E, p, \mathbf{r}\right) f(E, p, \mathbf{r}) \mathrm{d} E \mathrm{~d} p \mathrm{~d} \mathbf{r} .
$$




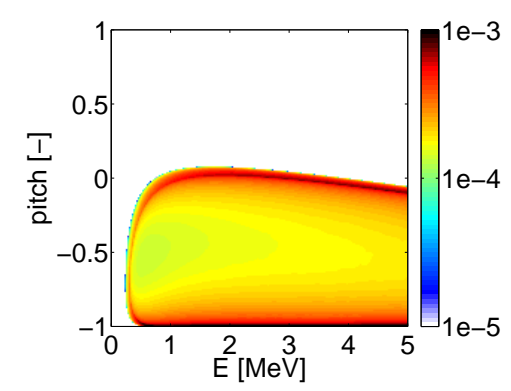

(a) $E_{n}=2 \mathrm{MeV}, \phi=45^{\circ}$.

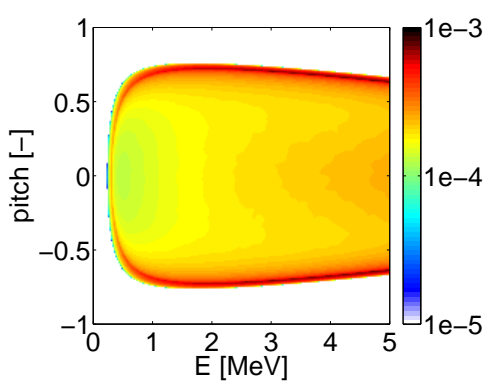

(d) $E_{n}=2 \mathrm{MeV}, \phi=90^{\circ}$.

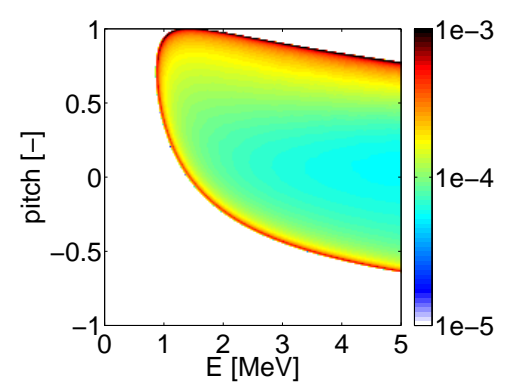

(b) $E_{n}=4 \mathrm{MeV}, \phi=45^{\circ}$.

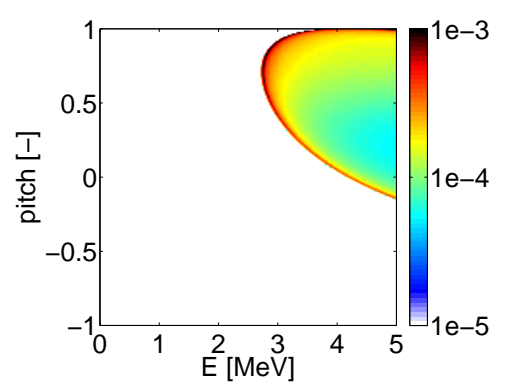

(c) $E_{n}=6 \mathrm{MeV}, \phi=45^{\circ}$.

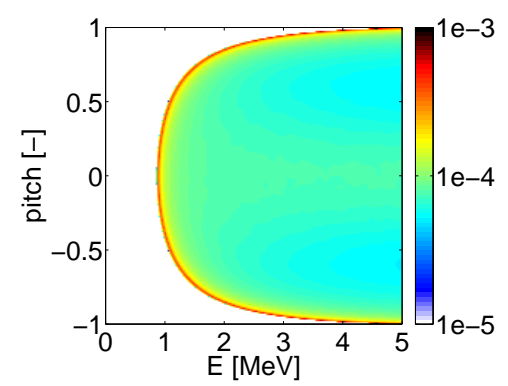

(e) $E_{n}=4 \mathrm{MeV}, \phi=90^{\circ}$.

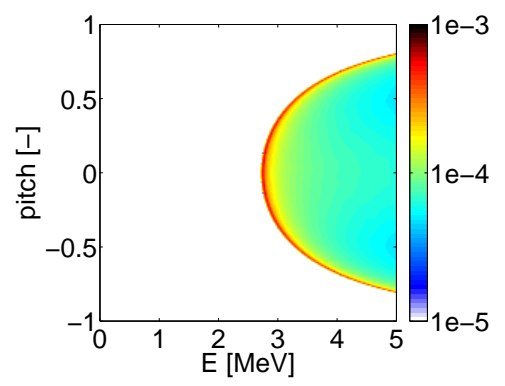

(f) $E_{n}=6 \mathrm{MeV}, \phi=90^{\circ}$.

FIG. 5: NES neutron energy weight functions in units of neutrons per second per fast ion.

Here $w_{\text {instrument }}\left(x_{1}, x_{2}, \phi, E, p, \mathbf{r}\right)$ is the instrument-specific weight function. As described in ${ }^{5}$ it is possible to calculate the instrument-specific weight functions:

$$
\begin{aligned}
w_{\text {instrument }}\left(x_{1}, x_{2}, \phi, E, p, \mathbf{r}\right)= & R(\phi, E, p, \mathbf{r}) \\
& \times \int_{0}^{\infty} \operatorname{pdf}_{E_{n}}\left(E_{n}, \phi, E, p\right) \mathcal{R}\left(x_{1}, x_{2}, E_{n}\right) \mathrm{d} E_{n},
\end{aligned}
$$

where $\mathcal{R}\left(x_{1}, x_{2}, E_{n}\right)$ is the instrumental response function, which gives the instrumental response in a range from $x_{1}$ to $x_{2}$, where $x$ is the specific measurement unit, as a function of the energy of the neutron.

Equation (4) can be understood as follows: The neutron rate $R$ determines the total number of neutrons produced in fusion reactions and emitted in the direction towards the detector per fast ion. The probability density function, $\operatorname{pdf}_{E_{n}}$, gives the probability that a neutron is produced in a certain energy range. The instrumental response function determines the signal which neutrons with this energy will produce in a measurement interval between $x_{1}$ and $x_{2}$. The integral over all neutron energies accounts for all neutron energies which might produce a signal between $x_{1}$ and $x_{2}$. A peaked instrumental response 
function, such as for TOFOR (figure 2a), ensures a good neutron energy resolution. On the other hand, in the case where $\mathcal{R}\left(x_{1}, x_{2}, E_{n}\right)$ is flat, independent of the neutron energy, the instrument-specific weight function can be approximated with the neutron rate times a constant:

$$
\begin{aligned}
w_{\text {instrument }}\left(x_{1}, x_{2}, \phi, E, p, \mathbf{r}\right)= & R(\phi, E, p, \mathbf{r}) \\
& \times \mathcal{R}\left(x_{1}, x_{2}\right) \int_{0}^{\infty} \operatorname{pdf}_{E_{n}}\left(E_{n}, \phi, E, p\right) \mathrm{d} E_{n} \\
= & R(\phi, E, p, \mathbf{r}) \mathcal{R}\left(x_{1}, x_{2}\right)
\end{aligned}
$$

since $\int_{0}^{\infty} \operatorname{pdf}_{E_{n}}\left(E_{n}, \phi, E, p\right) \mathrm{d} E_{n}=1$ by definition. In this case the instrument does not have any neutron energy resolution and is a neutron counter rather than a spectrometer. The compact spectrometers considered here have a relatively flat response function, but their step-function-like response functions ensure that they are still useful as spectrometers.

Equation (4) can be approximated in discrete form:

$$
\begin{aligned}
W_{\text {instrument }} & \simeq R \sum \operatorname{pdf}_{E_{n}} \mathcal{R} \Delta E_{n} \\
& \simeq R \sum \frac{\Delta \text { prob }}{\Delta E_{n}} \mathcal{R} \Delta E_{n} \\
& \simeq \sum W_{E_{n}} \mathcal{R},
\end{aligned}
$$

where $\operatorname{pdf}_{E_{n}}=\frac{\mathrm{d} \text { prob }}{\mathrm{d} E_{n}} \simeq \frac{\Delta \text { prob }}{\Delta E_{n}}$ has been used. $W_{E_{n}}$ is here the discrete neutron energy weight function calculated for a neutron energy interval $\Delta E_{n}$.

Figure 6 shows the time-of-flight weight functions calculated for TOFOR using the $90^{\circ}$ NES weight functions and the instrumental response function in figure 2a. Here we matched the flight times to the neutron energies by the maxima in the response function from figure $2 \mathrm{a}$, such that figures $5 \mathrm{~d}-5 \mathrm{f}$ should be compared with figures $6 \mathrm{a}-6 \mathrm{c}$, respectively. The very similar appearance of the TOFOR-specific and the fundamental NES weight functions indicate that the velocity-space sensitivity of TOFOR is almost as localized as it can possibly be. This is due to the peaked nature of the TOFOR response function.

Figure 7 shows the instrument-specific weight functions calculated for the NE213 liquid scintillator detector using the fundamental $45^{\circ}$ neutron energy weight functions and the response function in figure 2c. The light output values of 0.5 MeVee, 1.5 MeVee and 2.5 MeVee are chosen since they correspond to neutron energy detection thresholds of $2 \mathrm{MeV}, 4 \mathrm{MeV}$ and $6 \mathrm{MeV}$. The velocity-space sensitivity of the detector for low light output values is illustrated by a comparison of figures 5a and 7a. A detector with an ideal neutron energy 


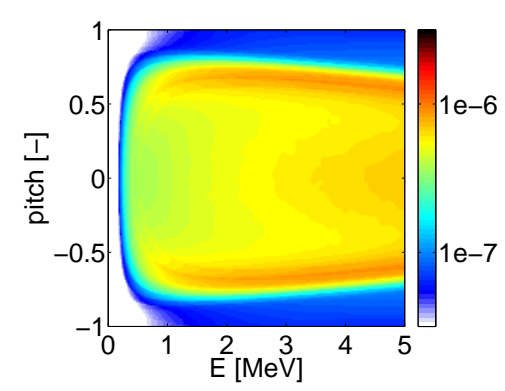

(a) Time-of-flight $=72$ ns.

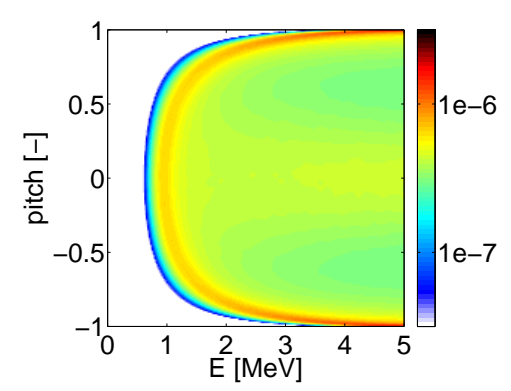

(b) Time-of-flight $=51$ ns.

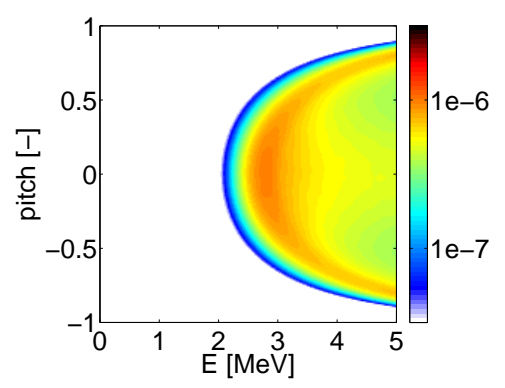

(c) Time-of-flight $=42 \mathrm{~ns}$.

FIG. 6: TOFOR specific weight functions in units of counts per bin per second per fast ion. These three time-of-flight values have been chosen since they correspond to neutron energies of $2 \mathrm{MeV}, 4 \mathrm{MeV}$ and $6 \mathrm{MeV}$, respectively.

resolution would be sensitive to ions with negative pitches and completely insensitive to ions with positive pitches (figure 4a). However, the NE213 detector measures almost the entire velocity space at low light output (figure 6a). This can also be realised from figure 2c since any neutron with an energy between $1.5 \mathrm{MeV}$ and $7 \mathrm{MeV}$ can produce a similar light output at 0.5 MeVee. Inspecting figures $5 \mathrm{~b}$ and $7 \mathrm{~b}$ it is seen how the low-energy boundary of the neutron energy weight function is retained in the instrument-specific weight function. However, some detailed internal features of the fundamental NES weight functions are masked by the instrument response. The same tendency is seen in figure $7 \mathrm{c}$.

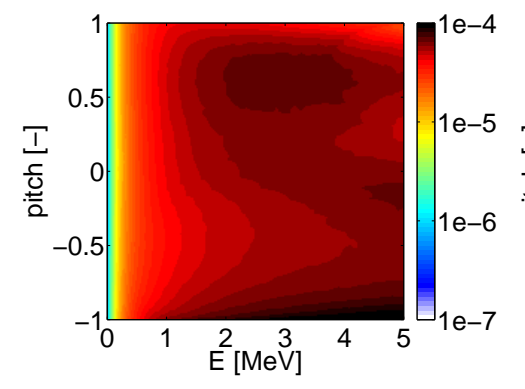

(a) Light output $=0.5 \mathrm{MeVee}$.

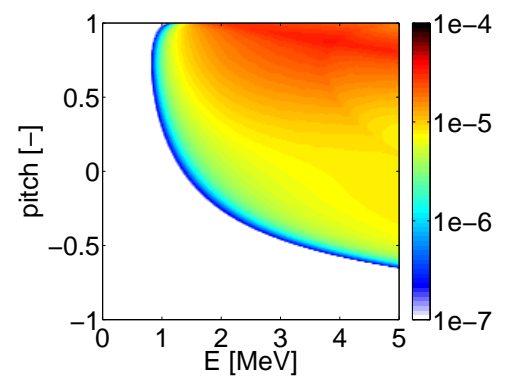

(b) Light output $=1.5$ MeVee.

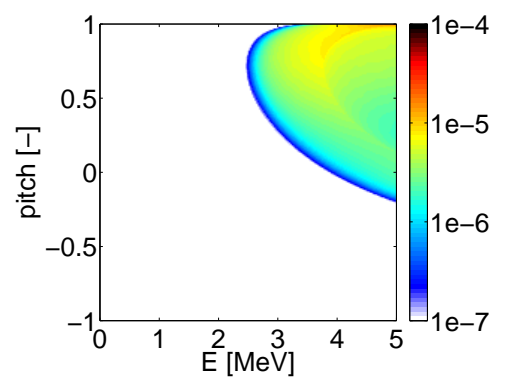

(c) Light output $=2.5$ MeVee.

FIG. 7: JET NE213 specific weight functions in units of neutrons per second per MeVee per fast ion. These light output values have been chosen since they correspond to threshold neutron energies of $2 \mathrm{MeV}, 4 \mathrm{MeV}$ and $6 \mathrm{MeV}$, respectively.

Figure 8 shows the instrument-specific weight functions calculated for the diamond de- 
tector. In deuterium plasmas, these are fairly similar to the weight functions for the NE213 detector since the instrumental response functions are also fairly similar.

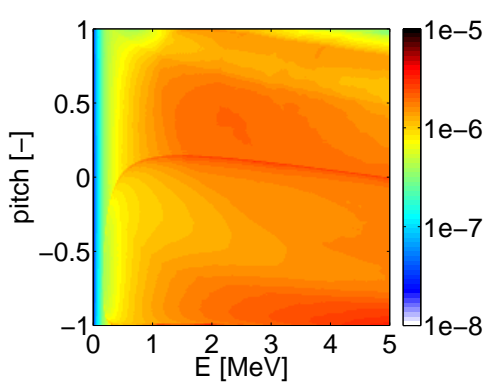

(a) $E_{d e p}=0.6 \mathrm{MeV}$.

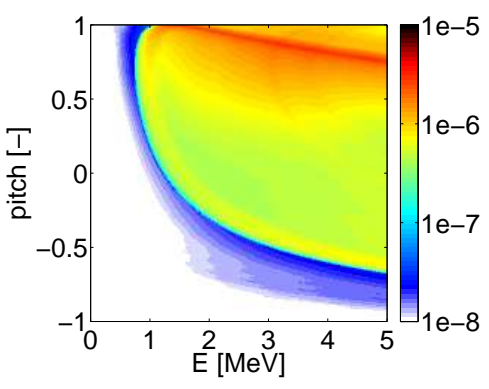

(b) $E_{\text {dep }}=1.1 \mathrm{MeV}$.

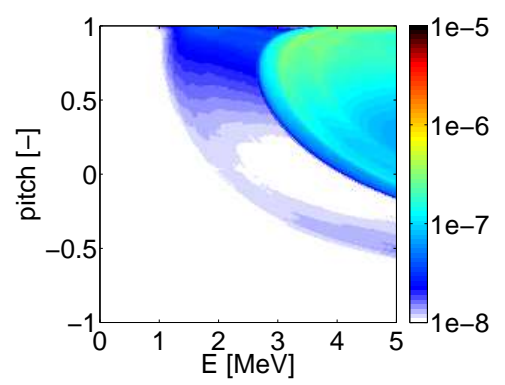

(c) $E_{\text {dep }}=1.7 \mathrm{MeV}$.

FIG. 8: Diamond specific weight functions in units of neutrons per second per MeV per fast ion calculated for three different deposited energy values. These three values have been chosen since they correspond to threshold neutron energy values of $2 \mathrm{MeV}, 4 \mathrm{MeV}$ and $6 \mathrm{MeV}$, respectively.

The instrument-specific weight functions calculated for the ASDEX Upgrade BC501A detector are shown in figure 9 . They are calculated using the fundamental $90^{\circ}$ neutron energy weight functions in figures 5d-5f and the ASDEX Upgrade BC501A instrument response function in figure 2d. The light output values of 0.5 MeVee, 1.5 MeVee and 3.0 MeVee are chosen since they correspond to neutron energy thresholds of $2 \mathrm{MeV}, 4 \mathrm{MeV}$ and $6 \mathrm{MeV}$, respectively. Once again the low light output weight function (figure 9a) is almost completely flat and therefore contains no velocity-space information of the fast ions. A measured light output of $1.5 \mathrm{MeVee}$ can only be produced by neutrons generated in fusion reactions involving an ion with an energy above $0.8 \mathrm{MeV}$ as shown in figure 9b. As seen in figure 9c, a light output of 3 MeVee can only be produced by neutrons from reactions involving an ion with an energy of more than $2.8 \mathrm{MeV}$ and pitch values between -0.8 and 0.8 . The weight functions are symmetric about pitch $=0$ because of the perpendicular view of the detector. A comparison of figures 9 and 7 illustrates the effect of changing the viewing geometry of a compact NES detector on the velocity-space sensitivity. 


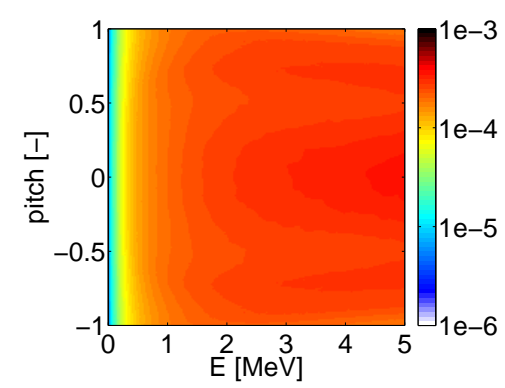

(a) Light output $=0.5 \mathrm{MeVee}$.

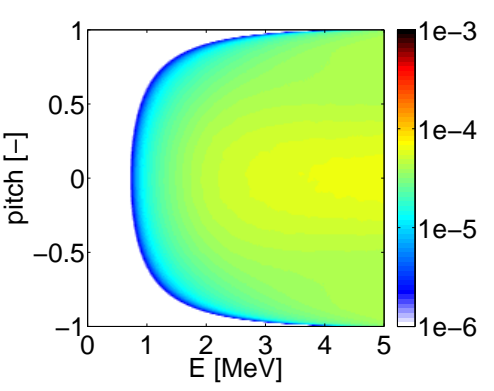

(b) Light output $=1.5$ MeVee.

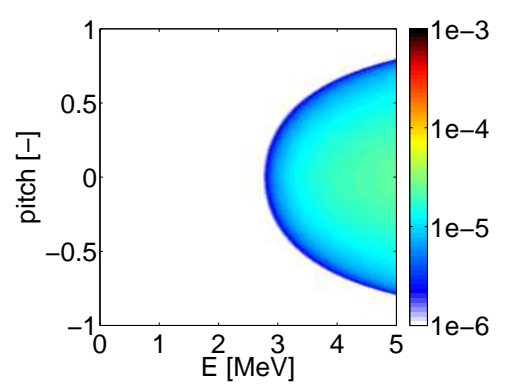

(c) Light output $=3.0$ MeVee.

FIG. 9: ASDEX Upgrade BC501A specific weight functions in units of neutrons per second per MeVee per fast ion. These light output values have been chosen since they correspond to threshold neutron energies of $2 \mathrm{MeV}, 4 \mathrm{MeV}$ and $6 \mathrm{MeV}$, respectively.

\section{DISCUSSION}

Here instrument-specific weight functions have been calculated for the most commonly used neutron spectrometers at JET for deuterium plasmas. In addition to these three, there exist several others. A magnetic proton recoil detector optimized for D-T neutrons shares the $45^{\circ}$ line of sight with the two compact detectors described here ${ }^{18}$. Another compact neutron spectrometer with a liquid organic scintillator is oriented horizontally with an angle of $25^{\circ}$ between its line of sight and the magnetic field ${ }^{19}$. Since it is based on the same principle it has a response function similar to $2 \mathrm{c}$, but the different angle changes the fundamental NES weight functions. The observable velocity-space region of this detector at large light output values will be even more restricted to ions with positive pitch. Finally, a diamond-based detector was recently installed sharing a line of sight with $\mathrm{TOFOR}^{28,29}$. In deuterium plasmas, the instrument-specific weight functions of this detector is obtained by multiplying the response function in figure $2 \mathrm{~b}$ and the fundamental weight functions in figures $5 \mathrm{~d}-5 \mathrm{f}$ and thus they will look very similar to the weight functions of the ASDEX Upgrade BC501A detector in figure 9. In deuterium-tritium plasmas, instead, the main neutron detection mechanism at play for diamond detectors and $14 \mathrm{MeV}$ neutrons is the ${ }^{12} \mathrm{C}(\mathrm{n}, \alpha){ }^{9} \mathrm{Be}$ reaction ${ }^{29,30}$, which produces a peak in the response function as for the MPR. Thus, in plasmas of deuteriumtritium, we may expect the velocity-space sensitivity of diamond-based detectors to more closely resemble that of the underlying fundamental weight functions, as for TOFOR in deuterium. This has been one of the motivations for the development of a second neutron 
spectrometer based on diamond detectors observing the plasma along a vertical sightline at JET. Another reason is that the NE213 and TOFOR find a more limited applicability in deuterium-tritium plasmas. Due to the 100 times larger neutron emission of $14 \mathrm{MeV}$ neutrons from these plasmas compared to deuterium, the NE213 detector may have to cope with intolerable MCounts/s counting rates. Similarly, TOFOR is not designed to offer a good spectroscopy performance at $14 \mathrm{MeV}$, both in terms of energy resolution and of signal/background ratio.

The fundamental neutron energy NES weight functions have already been used in analysing measurements at JET for identifying the observed region in velocity space ${ }^{9,10}$. Taking into account the instrumental response function improves the accuracy of this type of analysis substantially. Besides, instrument-specific weight functions as derived here may also serve as a parameter to choose among different neutron spectrometry concepts (time of flight, magnetic proton recoil, scintillation etc.) in their design phase. The figure of merit would here be the capability of a given instrumental concept to approach the velocity space sensitivity determined by the underlying fundamental weight functions shown in figure 5 .

Finally, the instrument-specific weight functions presented here allow us to go one step further. Equation (3) can be discretized and written as a matrix equation which can be solved as an inverse problem ${ }^{31-33}$.

$$
S=W_{\text {instrument }} F
$$

where $S, W_{\text {instrument }}$ and $F$ are discrete versions of $s, w_{\text {instrument }}$ and $f$, respectively. Equation (8) can be solved by tomography-like methods, see e.g. ${ }^{34-36}$ for more details. The instrumentspecific weight functions calculated here allow velocity-space tomography at $\mathrm{JET}^{37}$ combining NES measurements with gamma ray measurements for which the velocity-space weight functions were derived recently ${ }^{7,8}$ in a multi-diagnostic velocity-space tomography ${ }^{32,38,39}$. For ASDEX Upgrade the NES measurements might be used in a tomography together with measurements from the fast-ion $\mathrm{D}_{\alpha}$ and collective Thomson scattering diagnostics.

\section{CONCLUSION}

In this paper instrument-specific weight functions have been derived for the three main neutron spectrometers under operation in JET deuterium plasmas, as well as the neutron 
spectrometer at ASDEX Upgrade. Depending on the detection technique, instrumentspecific weight functions can approach, or not, the theoretical fundamental neutron emission weight functions. We find that the velocity sensitivity of TOFOR is close to the fundamental NES energy weight functions. For the NE213, diamond and BC501A detectors, instead, a localized velocity space sensitivity is preserved only at high light outputs and deposited energies. The instrument-specific weight functions derived here can help in benchmarking a specific spectrometer in its design phase by means of how localized its velocity-space sensitivity is. Finally, they are the essential prerequisite for a complete reconstruction of the fast ion energy distribution by means of tomographic inversions.

\section{ACKNOWLEDGEMENTS}

This work has been carried out within the framework of the EUROfusion Consortium and has received funding from the Euratom research and training programme 2014-2018 under grant agreement No 633053. The views and opinions expressed herein do not necessarily reflect those of the European Commission.

\section{REFERENCES}

${ }^{1}$ M. Salewski, S.K. Nielsen, H. Bindslev, V. Furtula, N.N. Gorelenkov, S.B. Korsholm, F. Leipold, F Meo, P.K. Michelsen, D. Moseev, and M. Stejner. On velocity space interrogation regions of fast-ion collective Thomson scattering at ITER. Nuclear Fusion, 51(8):083014, aug 2011.

${ }^{2}$ W W Heidbrink, Y Luo, K H Burrell, R W Harvey, R I Pinsker, and E Ruskov. Measurements of fast-ion acceleration at cyclotron harmonics using Balmer-alpha spectroscopy. Plasma Physics and Controlled Fusion, 49(9):1457-1475, sep 2007.

${ }^{3}$ M Salewski, B Geiger, D Moseev, W W Heidbrink, A S Jacobsen, S B Korsholm, F Leipold, J Madsen, S K Nielsen, J Rasmussen, M Stejner, and M Weiland. On velocityspace sensitivity of fast-ion D-alpha spectroscopy. Plasma Physics and Controlled Fusion, 56(10):105005, oct 2014.

${ }^{4}$ W W Heidbrink, J R Ferron, C T Holcomb, M A Van Zeeland, X Chen, C M Collins, A Garofalo, X Gong, B A Grierson, M Podestà, L Stagner, and Y Zhu. Confinement degra- 
dation by Alfvén-eigenmode induced fast-ion transport in steady-state scenario discharges. Plasma Physics and Controlled Fusion, 56(9):095030, 2014.

${ }^{5}$ A. S. Jacobsen, M. Salewski, J. Eriksson, G. Ericsson, A. Hjalmarsson, S. B. Korsholm, F. Leipold, S. K. Nielsen, J. Rasmussen, and M. Stejner. Velocity-space sensitivity of the time-of-flight neutron spectrometer at JETa). Review of Scientific Instruments, 85(11):11E103, nov 2014.

${ }^{6}$ A.S. Jacobsen, M. Salewski, J. Eriksson, G. Ericsson, S.B. Korsholm, F. Leipold, S.K. Nielsen, J. Rasmussen, and M. Stejner. Velocity-space sensitivity of neutron spectrometry measurements. Nuclear Fusion, 55(5):053013, may 2015.

${ }^{7}$ M. Salewski, M. Nocente, G. Gorini, A.S. Jacobsen, V.G. Kiptily, S.B. Korsholm, F. Leipold, J. Madsen, D. Moseev, S.K. Nielsen, J. Rasmussen, M. Stejner, and M Tardocchi. Velocity-space observation regions of high-resolution two-step reaction gamma-ray spectroscopy. Nuclear Fusion, 55(9):093029, sep 2015.

${ }^{8}$ M. Salewski, M. Nocente, G. Gorini, A.S. Jacobsen, V.G. Kiptily, S.B. Korsholm, F. Leipold, J. Madsen, D. Moseev, S.K. Nielsen, J. Rasmussen, M. Stejner, and M. Tardocchi. Fast-ion energy resolution by one-step reaction gamma-ray spectrometry. Nuclear Fusion, 56(4):046009, 2016.

${ }^{9}$ J. Eriksson, M. Nocente, F. Binda, C. Cazzaniga, S. Conroy, G. Ericsson, L. Giacomelli, G. Gorini, C. Hellesen, T. Hellsten, A. Hjalmarsson, A. S. Jacobsen, T. Johnson, V. Kiptily, T. Koskela, M. Mantsinen, M. Salewski, M. Schneider, S. Sharapov, M. Skiba, M. Tardocchi, and M. Weiszflog. Dual sightline measurements of $\mathrm{MeV}$ range deuterons with neutron and gamma-ray spectroscopy at JET. Nuclear Fusion, 55(12):123026, nov 2015.

${ }^{10}$ M. Schneider, T. Johnson, R. Dumont, J. Eriksson, L.-G. Eriksson, L. Giacomelli, J.-B. Girardo, T. Hellsten, E. Khilkevitch, V.G. Kiptily, T. Koskela, M. Mantsinen, M. Nocente, M. Salewski, S.E. Sharapov, and A.E. Shevelev. Modelling third harmonic ion cyclotron acceleration of deuterium beams for JET fusion product studies experiments. Nuclear Fusion, 56(11):112022, 2016.

${ }^{11}$ Xing Zhang, Zhongjing Chen, Xingyu Peng, Zhimeng Hu, Tengfei Du, Zhiqiang Cui, Xufei Xie, Xi Yuan, Tieshuan Fan, J. Kallne, G. Gorini, M. Nocente, M. Tardocchi, Liqun Hu, Guoqiang Zhong, Shiyao Lin, Baonian Wan, Xiangqing Li, Guohui Zhang, and Jinxiang Chen. Diagnosing NB plasmas on the EAST tokamak with new time-of-flight neutron spectrometer. Nuclear Fusion, 54(10):104008, oct 2014. 
${ }^{12} \mathrm{H}$ Tomita, H Iwai, T Iguchi, M Isobe, J Kawarabayashi, and C Konno. Development of neutron spectrometer toward deuterium plasma diagnostics in LHD. The Review of scientific instruments, 81(10):10D309, oct 2010.

${ }^{13}$ X Yuan, X Zhang, X Xie, G Gorini, Z Chen, X Peng, J Chen, G Zhang, T Fan, G Zhong, L Hu, and B Wan. Neutron energy spectrum measurements with a compact liquid scintillation detector on EAST. Journal of Instrumentation, 8(07):P07016-P07016, jul 2013.

${ }^{14}$ Yu.A. Kaschuck, B. Esposito, L.A. Trykov, and V.P. Semenov. Fast neutron spectrometry with organic scintillators applied to magnetic fusion experiments. Nuclear Instruments and Methods in Physics Research Section A: Accelerators, Spectrometers, Detectors and Associated Equipment, 476(1-2):511-515, jan 2002.

${ }^{15}$ M. Gatu Johnson, L. Giacomelli, A. Hjalmarsson, J. Källne, M. Weiszflog, E. Andersson Sundén, S. Conroy, G. Ericsson, C. Hellesen, E. Ronchi, H. Sjöstrand, G. Gorini, M. Tardocchi, A. Combo, N. Cruz, J. Sousa, and S. Popovichev. The 2.5-MeV neutron time-of-flight spectrometer TOFOR for experiments at JET. Nuclear Instruments and Methods in Physics Research Section A: Accelerators, Spectrometers, Detectors and Associated Equipment, 591(2):417-430, jun 2008.

${ }^{16}$ F. Binda, G. Ericsson, J. Eriksson, C. Hellesen, S. Conroy, and E. Andersson Sundén. Forward fitting of experimental data from a NE213 neutron detector installed with the magnetic proton recoil upgraded spectrometer at JETa). Review of Scientific Instruments, 85(11):11E123, 2014.

${ }^{17}$ C Cazzaniga, E Andersson Sundén, F Binda, G Croci, G Ericsson, L Giacomelli, G Gorini, E Griesmayer, G Grosso, G Kaveney, M Nocente, E Perelli Cippo, M Rebai, B Syme, and M Tardocchi. Single crystal diamond detector measurements of deuterium-deuterium and deuterium- tritium neutrons in Joint European Torus fusion plasmas. Review of Scientific Instruments, 3643(May):0-8, 2014.

${ }^{18}$ E. Andersson Sundén, H. Sjöstrand, S. Conroy, G. Ericsson, M. Gatu Johnson, L. Giacomelli, C. Hellesen, A. Hjalmarsson, E. Ronchi, M. Weiszflog, J. Källne, G. Gorini, M. Tardocchi, A. Combo, N. Cruz, A. Batista, R. Pereira, R. Fortuna, J. Sousa, and S. Popovichev. The thin-foil magnetic proton recoil neutron spectrometer MPRu at JET. Nuclear Instruments and Methods in Physics Research, Section A: Accelerators, Spectrometers, Detectors and Associated Equipment, 610(3):682-699, 2009. 
${ }^{19}$ Francesco Belli, Sean Conroy, Basilio Esposito, Luca Giacomelli, Vasily Kiptily, André Lücke, Daniele Marocco, Marco Riva, Helmut Schuhmacher, Brian Syme, Kai Tittelmeier, and Andreas Zimbal. Conceptual design, development and preliminary tests of a Compact Neutron Spectrometer for the JET experiment. IEEE transactions on nuclear science, 59(5):2512-2519, 2012.

${ }^{20}$ A. Zimbal, M. Reginatto, H. Schuhmacher, L. Bertalot, B. Esposito, F. Poli, J. M. Adams, S. Popovichev, V. Kiptily, and A. Murari. Compact NE213 neutron spectrometer with high energy resolution for fusion applications. Review of Scientific Instruments, 75(10 II):3553-3555, 2004.

${ }^{21}$ N. P. Hawkes, J. M. Adams, D. S. Bond, S. Croft, O. N. Jarvis, and N. Watkins. Measurements of the proton light output function of the organic liquid scintillator NE213 in several detectors. Nuclear Instruments and Methods in Physics Research, Section A: Accelerators, Spectrometers, Detectors and Associated Equipment, 476(1-2):190-194, 2002.

${ }^{22}$ M. Nocente, C. Cazzaniga, M. Tardocchi, F. Binda, J. Eriksson, L. Giacomelli, A. Muraro, M. Rebai, S. Sharapov, and G. Gorini. Fast ion energy distribution from third harmonic radio frequency heating measured with a single crystal diamond detector at the Joint European Torus. Review of Scientific Instruments, 86(10), 2015.

${ }^{23}$ L Giacomelli, A Zimbal, K Tittelmeier, H Schuhmacher, G Tardini, and R Neu. The compact neutron spectrometer at ASDEX Upgrade. The Review of scientific instruments, 82(12):123504, dec 2011.

${ }^{24}$ G Tardini, A Zimbal, B Esposito, F Gagnon-Moisan, D Marocco, R Neu, H Schuhmacher, and the Asdex Upgrade Team. First neutron spectrometry measurements in the ASDEX Upgrade tokamak. Journal of Instrumentation, 7(03):C03004-C03004, mar 2012.

${ }^{25}$ M. Rebai, L. Giacomelli, A. Milocco, M. Nocente, D. Rigamonti, M. Tardocchi, F. Camera, C. Cazzaniga, Z.J. Chen, T.F. Du, T.S. Fan, A. Giaz, Z.M. Hu, T. Marchi, X.Y. Peng, and G. Gorini. Response function of single crystal synthetic diamond detectors to $1-4 \mathrm{MeV}$ neutrons for spectroscopy of D plasmas. Review of Scientific Instruments, 87(11), 2016.

${ }^{26}$ G. Tardini, F. Gagnon-Moisan, and A. Zimbal. Characterisation of a BC501A compact neutron spectrometer for fusion research. Review of Scientific Instruments, 87(10):103504, 2016.

${ }^{27}$ Binda, F. and Eriksson, J. and Ericsson, G. and Hellesen, C. and Conroy, S. and Nocente, M. and Sundén, E. Andersson. Generation of the neutron response function of an NE213 
scintillator for fusion applications. Nuclear Instruments and Methods in Physics Research Section A: Accelerators, Spectrometers, Detectors and Associated Equipment, In press , 2017.

${ }^{28}$ A. Muraro, L. Giacomelli, M. Nocente, M. Rebai, D. Rigamonti, F. Belli, P. Calvani, J. Figueiredo, M. Girolami, G. Gorini, G. Grosso, A. Murari, S. Popovichev, D. M. Trucchi, and M. Tardocchi. First neutron spectroscopy measurements with a pixelated diamond detector at JET. Review of Scientific Instruments, 87(11):11D833, 2016.

${ }^{29}$ C. Cazzaniga, M. Nocente, M. Rebai, M. Tardocchi, P. Calvani, G. Croci, L. Giacomelli, M. Girolami, E. Griesmayer, G. Grosso, M. Pillon, D. M. Trucchi, and G. Gorini. A diamond based neutron spectrometer for diagnostics of deuterium-tritium fusion plasmas. Review of Scientific Instruments, 85(11):11E101, 2014.

${ }^{30}$ L. Giacomelli, M. Nocente, M. Rebai, D. Rigamonti, A. Milocco, M. Tardocchi, Z. J. Chen, T. F. Du, T. S. Fan, Z. M. Hu, X. Y. Peng, A. Hjalmarsson, and G. Gorini. Neutron emission spectroscopy of DT plasmas at enhanced energy resolution with diamond detectors. Review of Scientific Instruments, 87(11):1-5, 2016.

${ }^{31}$ M. Salewski, B. Geiger, S.K. Nielsen, H. Bindslev, M. García-Muñoz, W.W. Heidbrink, S.B. Korsholm, F. Leipold, F. Meo, P.K. Michelsen, D. Moseev, M. Stejner, and G. Tardini. Tomography of fast-ion velocity-space distributions from synthetic CTS and FIDA measurements. Nuclear Fusion, 52(10):103008, oct 2012.

${ }^{32}$ M. Salewski, B. Geiger, S.K. Nielsen, H. Bindslev, M. García-Muñoz, W.W. Heidbrink, S.B. Korsholm, F. Leipold, J. Madsen, F. Meo, P.K. Michelsen, D. Moseev, M. Stejner, and G. Tardini. Combination of fast-ion diagnostics in velocity-space tomographies. Nuclear Fusion, 53(6):063019, jun 2013.

${ }^{33}$ M. Salewski, B. Geiger, A.S. Jacobsen, M. García-Muñoz, W.W. Heidbrink, S.B. Korsholm, F. Leipold, J. Madsen, D. Moseev, S.K. Nielsen, J. Rasmussen, M. Stejner, G. Tardini, and M. Weiland. Measurement of a 2D fast-ion velocity distribution function by tomographic inversion of fast-ion D-alpha spectra. Nuclear Fusion, 54(2):023005, feb 2014. ${ }^{34}$ A S Jacobsen, L Stagner, M Salewski, B Geiger, W W Heidbrink, S B Korsholm, F Leipold, S K Nielsen, J Rasmussen, M Stejner, H Thomsen, and M Weiland. Inversion methods for fast-ion velocity-space tomography in fusion plasmas. Plasma Physics and Controlled Fusion, 58(4):045016, apr 2016. 
${ }^{35}$ M Weiland, B Geiger, A S Jacobsen, M Reich, M Salewski, and T Odstrčil. Enhancement of the FIDA diagnostic at ASDEX Upgrade for velocity space tomography. Plasma Physics and Controlled Fusion, 58(2):025012, 2016.

${ }^{36} \mathrm{M}$ Salewski, B Geiger, A S Jacobsen, P C Hansen, W W Heidbrink, S B Korsholm, F Leipold, J Madsen, D Moseev, S K Nielsen, M Nocente, T Odstrčil, J Rasmussen, L Stagner, M Stejner, and M Weiland. High-definition velocity-space tomography of fastion dynamics. Nuclear Fusion, 56, 2016.

${ }^{37}$ M. Salewski, M. Nocente, A.S. Jacobsen, F. Binda, C. Cazzaniga, G. Ericsson, J. Eriksson, G. Gorini, C. Hellesen, A. Hjalmarsson, V.G. Kiptily, T. Koskela, S.B. Korsholm, T. KurkiSuonio, F. Leipold, J. Madsen, D. Moseev, S.K. Nielsen, J. Rasmussen, M. Schneider, S.E. Sharapov, M. Stejner, M. Tardocchi, and JET Contributors. Mev-range velocity-space tomography from gamma-ray and neutron emission spectrometry measurements at jet. Nuclear Fusion, 57(5):056001, 2017.

${ }^{38}$ A S Jacobsen, M Salewski, B Geiger, S B Korsholm, F Leipold, S K Nielsen, J Rasmussen, M Stejner, and M Weiland. Benchmark and combined velocity-space tomography of fast-ion D-alpha spectroscopy and collective Thomson scattering measurements. Plasma Physics and Controlled Fusion, 58(4):042002, apr 2016.

${ }^{39}$ J. Rasmussen, S.K. Nielsen, M. Stejner, J. Galdon-Quiroga, M. Garcia-Munoz, B. Geiger, A.S. Jacobsen, F. Jaulmes, S.B. Korsholm, N. Lazanyi, F. Leipold, F. Ryter, M. Salewski, M. Schubert, J. Stober, D. Wagner, the ASDEX Upgrade Team, and the EUROFusion MST1 Team. Collective Thomson scattering measurements of fast-ion transport due to sawtooth crashes in ASDEX Upgrade. Nuclear Fusion, 56(11):112014, 2016.

${ }^{40} \mathrm{H}$ Zohm for the ASDEX Upgrade Team and the EUROfusion MST1 Team. Recent ASDEX Upgrade research in support of ITER and DEMO. Nuclear Fusion, 55(10):104010, 2015. 